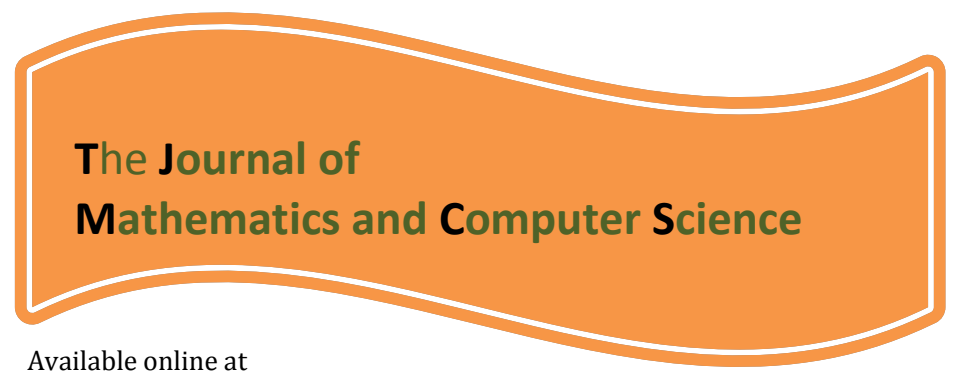

http://www.TJMCS.com

The Journal of Mathematics and Computer Science Vol. 4 No.3 (2012) 371 - 379

\title{
Analytical Solution for Nnonlinear Vibration of Micro-Electro- mechanical System (MEMS) by Frequency-Amplitude Formulation Method
}

\author{
M. Mashinchi Joubari ${ }^{1}$, R. Asghari ${ }^{2}$ \\ ${ }^{1}$ Department of Mechanical Engineering, Babol University of Technology, Babol, Iran \\ mmmjouybari@gmail.com \\ ${ }^{2}$ Applied Mathematics Department, Mathematics Science Faculty, Guilan University, Rasht, Iran \\ meisam.mathhome@gmail.com
}

Received: February 2012, Revised: May 2012

Online Publication: July 2012

\begin{abstract}
In this paper, one analytical technique named Frequency-Amplitude Formulation Method (FAF) isused to obtain the behavior and frequency of theelectrostatically actuated microbeams. The main aim of the work is obtaining highly accurate analytical solution for nonlinear free vibration of a microbeam and investigates the dynamic behavior of the system. Results reveal that the nonlinear frequency of oscillatory system remarkably affected with the initial conditions. In contrast to the time marching solution results, the present analytical method is effective and convenient. It is predictable that the FAF can apply for various problems in engineering specially vibration equations.
\end{abstract}

\section{Introduction}

Micro-electro-mechanical systems (MEMS) are batch-fabricated devices and structures at a microscale level [1]. Since its inception, MEMS technologies are of tremendous importance in various engineering fields. Because of its small size, low power consumption and high reliability, we have seen many potential applications of MEMS actuators and sensors in aerospace, optical and biomedical engineering [2-4]. Generally, an archetypal electrostatic micro-switch is one of the significant MEMS devices, which can be modeled by an electrostatically driven microbeam and a pair of fixed electrodes. From a physical point of view, the dynamical motion of the microbeam can be governed by the electrostatic as well as intermolecular forces [5]. Due to a voltage difference between the electrode and the microbeam, the electrostatic actuation is created by the induced 
electrostatic charges. Besides, the intermolecular force accounts for the molecular interaction of the tiny gap size between the electrode and the microbeam.

In order to advance knowledge in micro/nanotechnology, the analysis of dynamic and stability responses of various engineering models [5-12] has thus attracted intensive research attention. Recently, Fu et al. [13] investigated the nonlinear oscillation problem arising in the MEMS microbeam model by means of the energy balance method. Because this problem [13] is strongly nonlinear when subjecting to large amplitudes of motion and physical parameters, so it is hardly amenable to analytically obtain an exact solution for such a problem.

Almost all oscillation problems in engineering are modeled by nonlinear differential equations. Obtaining exact solution for these nonlinear problems is a great propose but, in most cases it is difficult to achieve them. Therefore special techniques should be applied to solve them. Many of these techniques have been performed in recent literatures such as Homotopy Perturbation Method (HPM) [14-18], Homotopy Analysis Method (HAM) [19-21], Iteration Perturbation Method (IPM) [22-24], Variational Iteration Method (VIM) [25-28], Differential Transformation Method (DTM) [29-30], Frequency Amplitude Formulation (FAF) [31-33], Max-Min Approach (MMA) [3438].

\section{Mathematical model and solution approach}

Consider a fully clamped microbeam with uniform thickness $\mathrm{h}$, length $\mathrm{l}$, width $\mathrm{b}(b \geq 5 h)$,effective modulus $\bar{E}=\frac{E}{1-v^{2}}$, Young's modulus E, Poisson's ratio $\mathrm{m}$ and density q, as shown in Fig. 1. Employing theclassical beam theory and taking into account of the mid-plane stretching effect as well as the distributedelectrostatic force, the following dimensionless equation of motion for the microbeam can be formulated via the Galerkin method [13].

$\ddot{u}\left(a_{1} u^{4}+a_{2} u^{2}+a_{3}\right)+a_{4} u+a_{5} u^{3}+a_{6} u^{5}+a_{7} u^{7}=0, u(0)=A, \dot{u}(0)=0$

where $u$ is thedimensionless deflection of the microbeam, a dot denotes the derivative with respect to the dimensionless time variable $\bar{t}=t \sqrt{\frac{\bar{E} I}{\rho b h l^{4}}}$ with I and $t$ being the second moment of area of the beam cross-section and time, respectively.The expressions of the governing parameters $a_{i}(i=1-7)$ can be written as:

$$
\begin{aligned}
& a_{1}=\int_{0}^{1} \phi^{6} d \zeta, a_{2}=-2 \int_{0}^{1} \phi^{4} d \zeta, a_{3}=\int_{0}^{1} \phi^{2} d \zeta \\
& a_{4}=\int_{0}^{1}\left(\phi^{\prime \prime \prime} \phi-N \phi^{\prime \prime} \phi-V^{2} \phi^{2}\right) d \zeta \\
& a_{5}=-\int_{0}^{1}\left(2 \phi^{\prime \prime \prime} \phi^{3}-2 N \phi^{\prime \prime} \phi^{3}+\alpha \phi^{\prime \prime} \phi \int_{0}^{1} \phi^{\prime 2} d \zeta\right) d \zeta \\
& a_{6}=\int_{0}^{1}\left(\phi^{\prime \prime \prime \prime} \phi^{5}-N \phi^{\prime \prime} \phi^{5}+2 \alpha \phi^{\prime \prime} \phi^{3} \int_{0}^{1} \phi^{\prime 2} d \zeta\right) d \zeta
\end{aligned}
$$


$a_{7}=-\int_{0}^{1}\left(\alpha \phi^{\prime \prime} \phi^{5} \int_{0}^{1} \phi^{2} d \zeta\right) d \zeta$

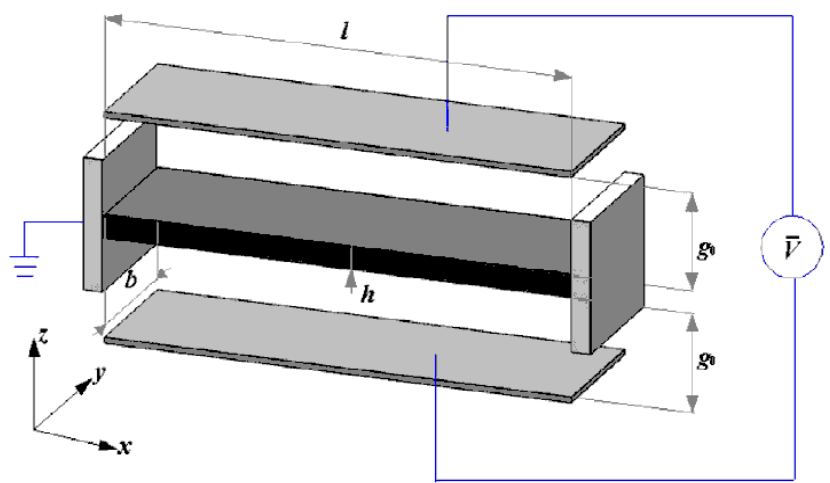

Fig. 1. Geometry for an electrostatically actuated microbeam with fixed supports

\section{Application of frequency amplitude formulation}

For solving Eq.(1) with frequency amplitude formulation, we use the trial functions $u_{1}(t)=A \cos (t)$ and $u_{2}(t)=A \cos (\omega t)$, which are the solutions of the following linear equations, respectively,

$\ddot{u}+\varpi_{1}^{2} u=0, \varpi_{1}^{2}=1$

$\ddot{u}+\varpi_{2}^{2} u=0, \varpi_{2}^{2}=\omega^{2}$

The residuals are:

$$
\begin{aligned}
& R_{1}(t)=\left(-a_{3} A+a_{4} A\right) \cos t+\left(-a_{2} A^{3}+a_{5} A^{3}\right) \cos ^{3} t+ \\
& \left(-a_{1} A^{5}+a_{6} A^{5}\right) \cos ^{5} t+a_{7} A^{7} \cos ^{7} t \\
& R_{2}(t)=\left(-a_{3} A+a_{4} A\right) \cos \omega t+\left(-a_{2} A^{3}+a_{5} A^{3}\right) \cos ^{3} \omega t+ \\
& \left(-a_{1} A^{5}+a_{6} A^{5}\right) \cos ^{5} \omega t+a_{7} A^{7} \cos ^{7} \omega t
\end{aligned}
$$

We introduce two new residual variables $\tilde{R}_{1}$ and $\tilde{R}_{2}$ define as[31-33]:

$$
\begin{aligned}
& \tilde{R}_{1}=\frac{4}{T_{1}} \int_{0}^{\frac{T_{1}}{4}} R_{1}(t) \cos t d t, \quad T_{1}=2 \pi \\
& \tilde{R}_{2}=\frac{4}{T_{2}} \int_{0}^{\frac{T_{2}}{4}} R_{2}(t) \cos \omega t d t, \quad T_{1}=\frac{2 \pi}{\omega}
\end{aligned}
$$

we can approximately determine $\omega^{2}$ in the form,

$$
\omega^{2}=\frac{\tilde{R}_{2}-\omega^{2} \tilde{R}_{1}}{\tilde{R}_{2}-\tilde{R}_{1}}
$$

For the Eq.(1), by a simple calculation,we obtain: 
$\tilde{R}_{1}=\frac{64\left(-a_{3} A+a_{4} A\right)+48\left(-a_{2} A^{3}+a_{5} A^{3}\right)+40\left(-a_{1} A^{5}+a_{6} A^{5}\right)+35 A^{7} a_{7}}{128}$

and

$\tilde{R}_{2}=\frac{64\left(-a_{3} \omega^{2} A+a_{4} A\right)+48\left(-a_{2} \omega^{2} A^{3}+a_{5} A^{3}\right)+40\left(-a_{1} \omega^{2} A^{5}+a_{6} A^{5}\right)+35 A^{7} a_{7}}{128}$

Applying Eq.(9), we have:

$\omega=\omega_{F A F}=\sqrt{\frac{-b-\sqrt{b^{2}-4 a c}}{2 a}}$

Where $\mathrm{a}, \mathrm{b}$ and $\mathrm{c}$ are as follows:

$a=-64 a_{3} A-48 a_{2} A^{3}-40 a_{1} A^{5}$

$b=64 a_{3} A+64 a_{4} A+48 a_{2} A^{3}+48 a_{5} A^{3}+40 a_{1} A^{5}+40 a_{6} A^{5}+35 A^{7} a_{7}$

$c=-64 a_{4} A-48 a_{5} A^{3}-40 a_{6} A^{5}-35 A^{7} a_{7}$

The periodic solution as follows:

$u(t)=A \cos \left(\omega_{F A F} t\right)=A \cos \left(\sqrt{\frac{-b-\sqrt{b^{2}-4 a c}}{2 a}} t\right)$

\section{Results}

In this section the result which obtained using the FAFis compared with the time marching results for the following parameters.

$A=0.3, N=10, \alpha=24$

Figures 2 and 3 show the displacement of the microbeam(u(t))with FAF, EBM [13] and ODE-solver in MATLAB. Also, comparison between frequencies obtained by FAF and EBM are illustrated in figure 4 .

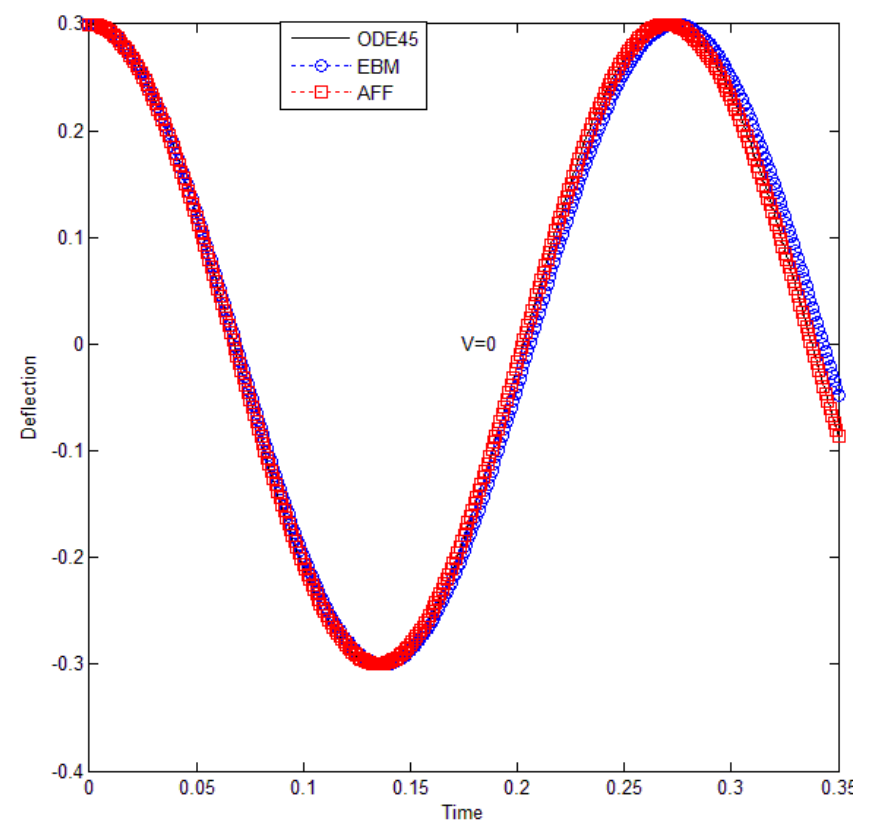

Fig2.Comparison between the FAF, EBM and ODE45 results of the tapered beam ( $A=0.3, V=0$ ). 
Figure 5 illustrates the error percentages of the analytical solution used in this work and presented in [13]. As time passes the FAF's error decrease with respect to the other analytical result. The error percentage of the analytical solutions results calculated from the following equation.

$\%$ Error $=\frac{\left|u_{\text {Exact }}-u_{\text {Analytical }}\right|}{u_{\text {Exact }}} \times 100$

Figure 6 shows the phase-plane of the microbeam for $\mathrm{V}=0$ and $A=0.3$. In this figure the FAF and EBM compared with ODE45 solution. Moreover, Figure 7 shows the vibration behavior of the MEMS for different initial amplitudes.

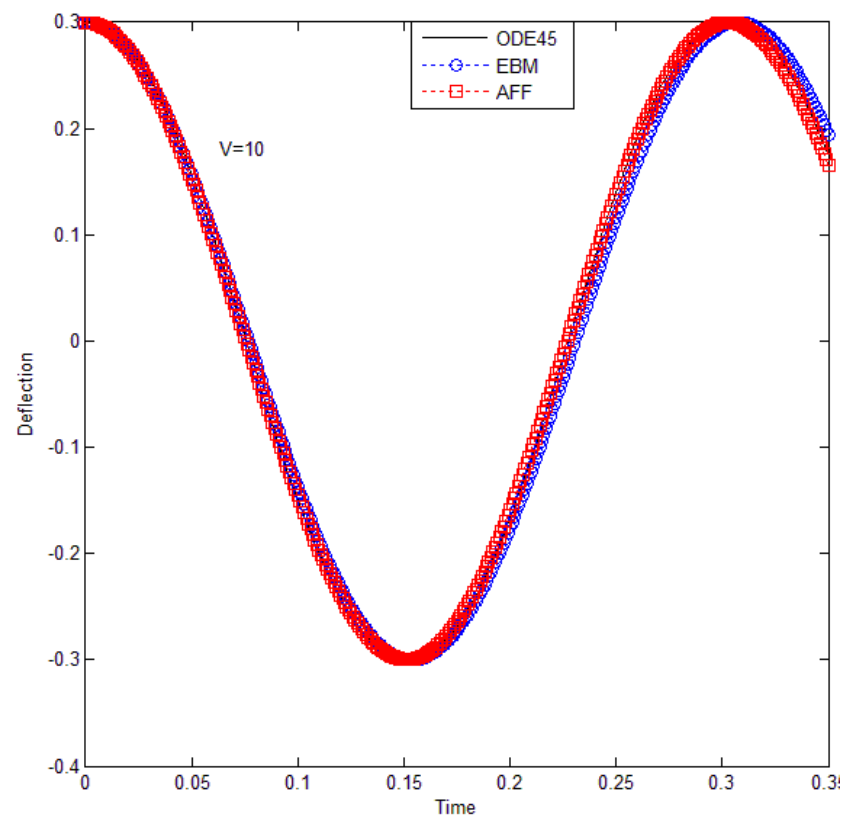

Fig3.Comparison between the FAF, EBM and ODE45 results of the tapered beam $(A=0.3, V=10)$. 
M. Mashinchi Joubari, R. Asghari/ TJMCS Vol. 4 No. 3 (2012) 371 - 379

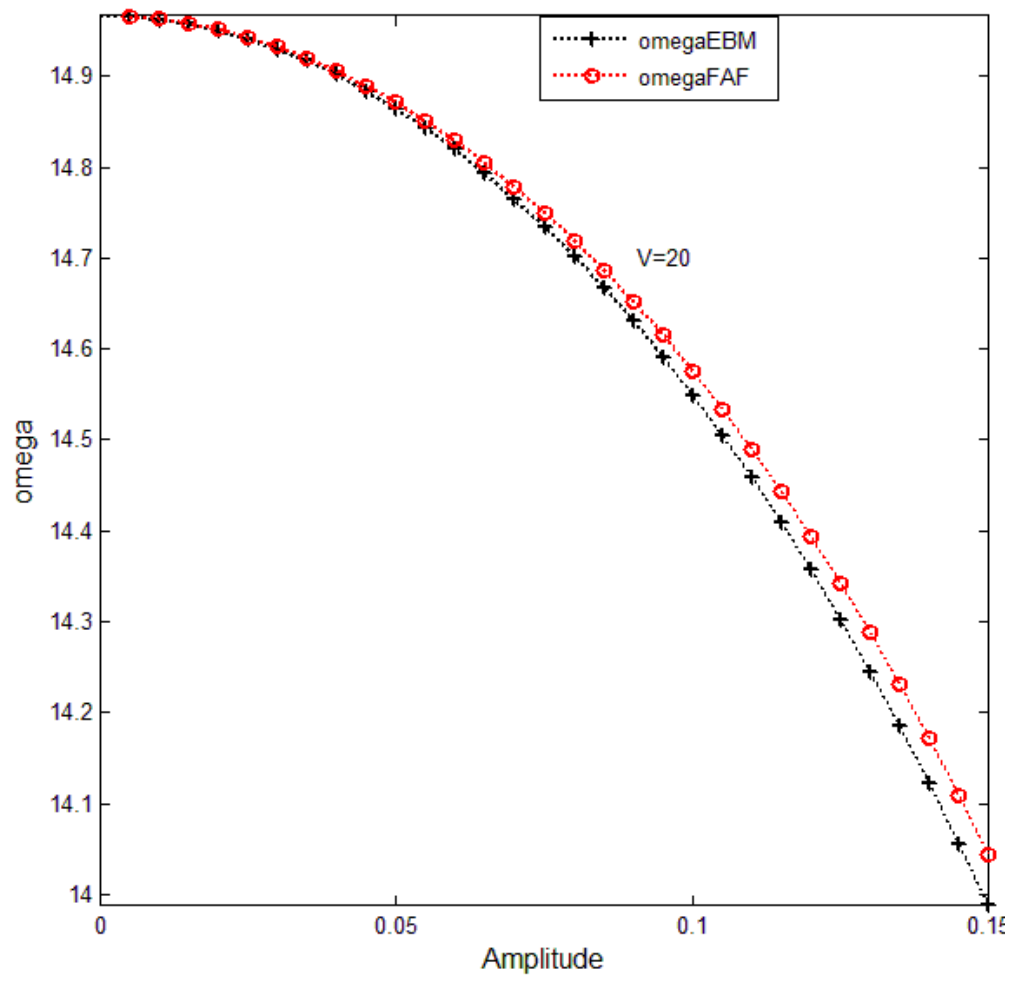

Fig 4. Nonlinear frequency versus amplitude for various $V=20$

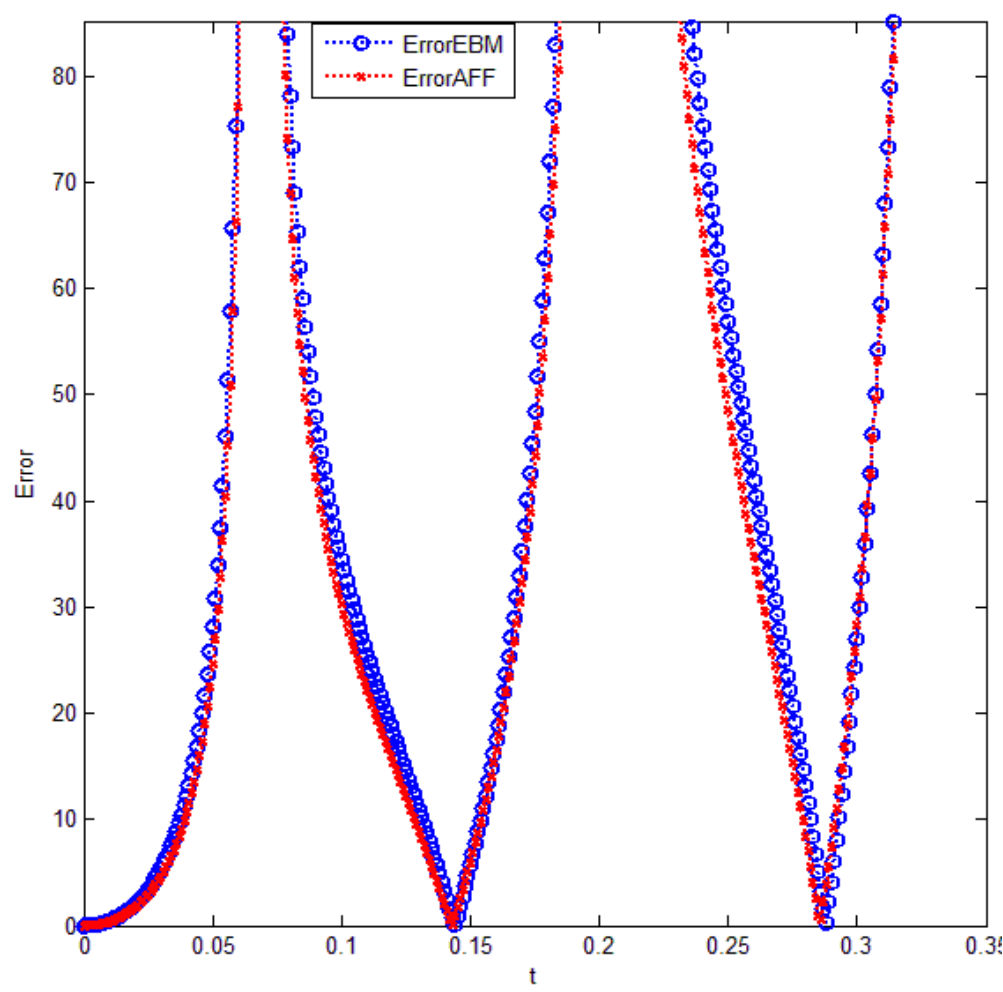

Fig 5. Error percentage for FAF and other analytical solution( $A=0.3, V=0)$. 


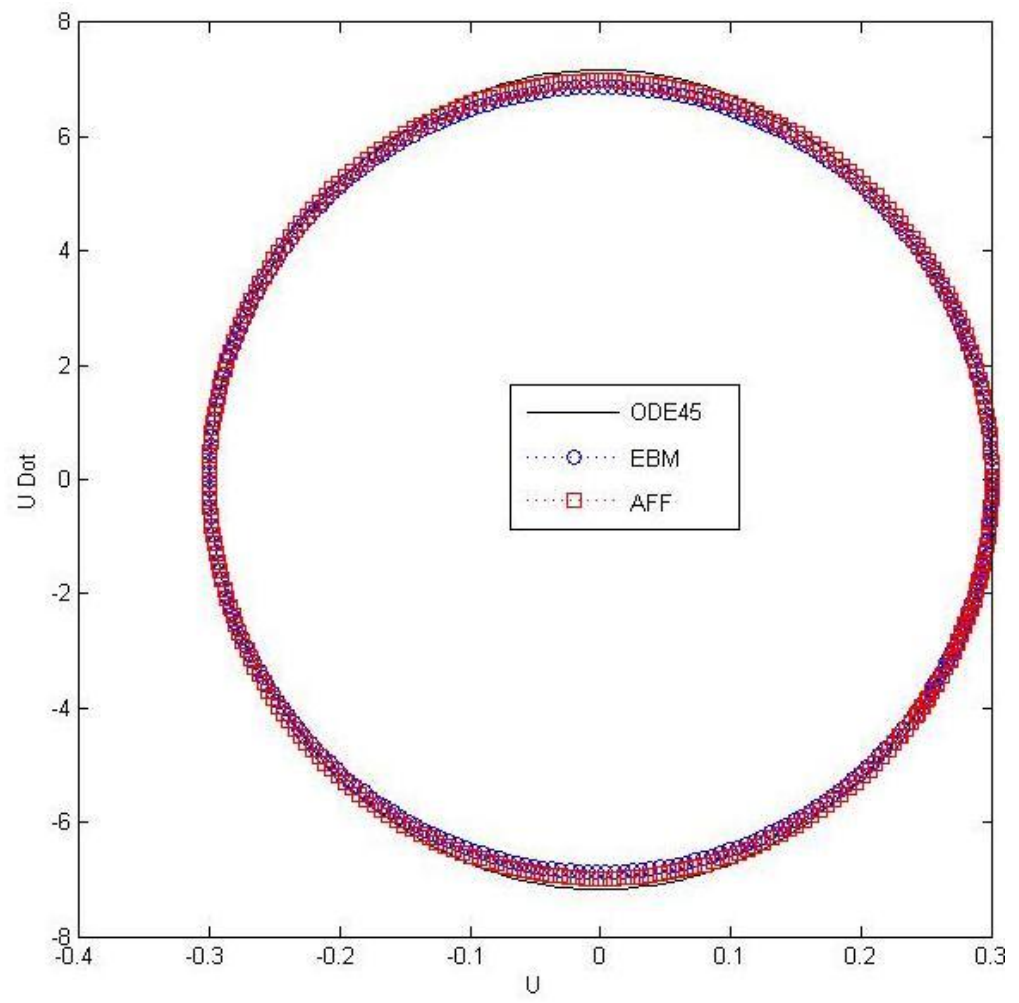

Fig 6. Phase plane of the system using exact solution and analytical solutions ( $A=0.3, V=0$ )

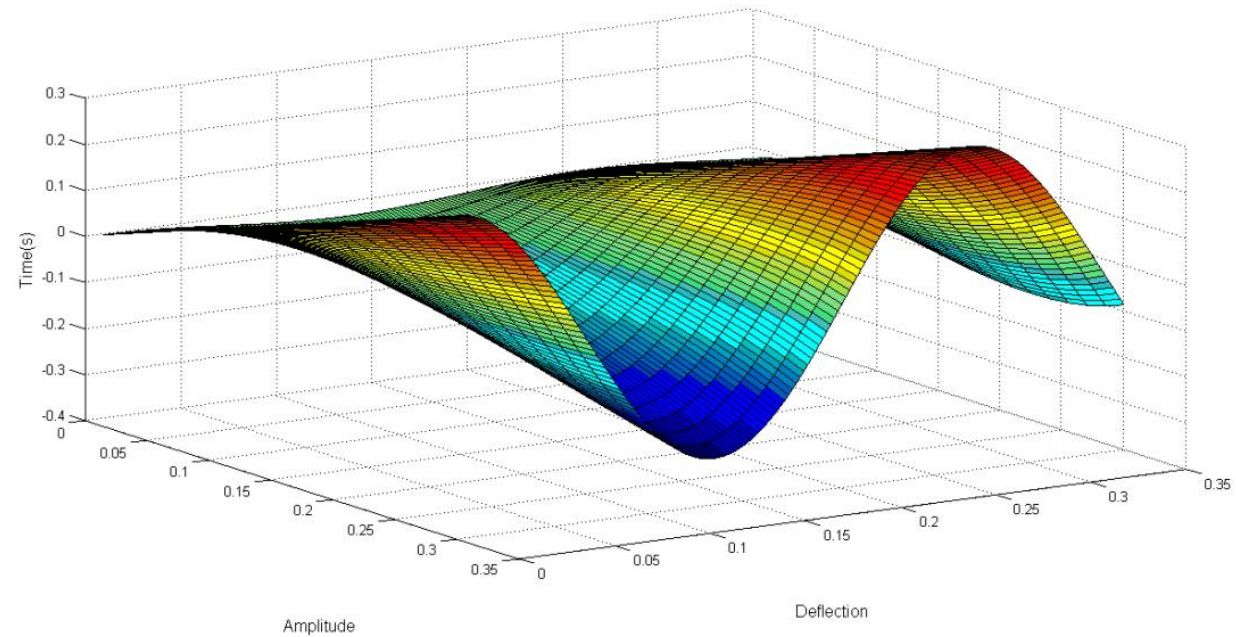

Fig 7. The vibration behavior of the tapered beam $A=0.3, V=10$

\section{Conclusions}

In this paper, the FAF was employed to solve the governing equation of nonlinear oscillations of microbeams. The results of FAF have excellent agreement with the results obtained by EBM and ODE45solution. The error percentage achieved by FAF decreased during the time against of the other analytical solution. This method is simple and doesn't need to programming but it is important to choose the correct frequency for solving some complicated problems. It can be 


\section{Mashinchi Joubari, R. Asghari/ TJMCS Vol. 4 No. 3 (2012) 371 - 379}

approved that FAF is powerful and efficient technique in finding analytical solutions for a wide classes of nonlinear oscillator.

\section{References}

[1] Lyshevski SE. MEMS and NEMS: systems, devices and structures. Boca Raton: CRC Press; 2002.

[2] Osiander R, Ann Garrison Darrin M, Champion JL, editors. MEMS and microstructures in aerospace applications. Boca Raton: Taylor \& Francis; 2006.

[3] Abeysinghe DC, Dasgupta S, Boyd JT, Jackson HE. A novel MEMS pressure sensor fabricated on an optical fiber. IEEE Photon TechnolLett 2001;13:993-5.

[4] Jain A, Goodson KE. Thermal microdevices for biological and biomedical applications. J ThermBiol 2011;36:209-18.

[5] Jia XL, Yang J, Kitipornchai S, Lim CW. Free vibration of geometrically nonlinear micro-switches under electrostatic and Casimir forces. Smart MaterStruct 2010;19. Article No. 115028.

[6] Lee SI, Howell SW, Raman A, Reifenberger R, Nguyen CV, Meyyappan M. Nonlinear tapping dynamics of multi-walled carbon nanotube tipped atomicforce microcantilevers. Nanotechnology 2004;15:416-21.

[7] Batra RC, Porfiri M, Spinello D. Electromechanical model of electrically actuated narrow microbeams. J MicroelectromechSyst 2006;15:1175-89.

[8] Rhoads JF, Shaw SW, Turner KL. The nonlinear response of resonant microbeam systems with purelyparametric electrostatic actuation. J MicromechMicroeng 2006;16:890-9.

[9] MoghimiZand M, Ahmadian MT. Application of homotopy analysis method in studying dynamic pull-in instability of microsystems. Mech ResCommun 2009;36:851-8.

[10] MoghimiZand M, Ahmadian MT. Vibrational analysis of electrostatically actuated microstructures considering nonlinear effects. Commun NonlinearSciNumerSimul 2009;14:1664-78.

[11] Demir Ç, Civalek Ö, Akgöz B. Free vibration analysis of carbon nanotubes based on shear deformable beam theory by discrete singular convolutiontechnique. Math ComputAppl 2010;15:57-65.

[12] Akgöz B, Civalek Ö. Application of strain gradient elasticity theory for buckling Analysis of protein microtubules. CurrApplPhys 2011;11:1133-8.

[13] Fu YM, Zhang J, Wan LJ. Application of the energy balance method to a nonlinear oscillator arising in the microelectromechanical system (MEMS). CurrApplPhys 2011;11:482-5.

[14] Fereidoon, A., Ganji, D.D., Kaliji, H.D., Ghadimi, M.: Analytical solution for vibration of buckled beams. International Journal of Research and Reviews in Applied Sciences, 4(3), 17-21 (2010)

[15] Nawaz, Yasir: Variational iteration method and homotopy perturbation method for fourth-order fractional integro-differential equations. Computers and Mathematics with Applications 61, 2330-2341 (2011)

[16] Ganji, S.S., Barari, A., Sfahani, M.G., Domairry, G., TeimourzadehBaboli, P.: Consideration of transient stream/aquifer interaction with the nonlinear Boussinesq equation using HPM. Journal of King Saud University-Science 23, 211-216 (2011)

[17] Farrokhzad, F., Mowlaee, P., Barari, A., Choobbasti, A.J., Kaliji, H.D.: Analytical investigation of beam deformation equation using perturbation, homotopy perturbation, variational iteration and optimal homotopy asymptotic methods. Carpathian Journal of Mathematics 27(1), 51-63 (2011)

[18] Chun Changbum, SakthivelRathinasamy: Homotopy perturbation technique for solving two-point boundary value problems-comparison with other methods. Computer Physics Communications 181, 10211024 (2010)

[19] Fooladi, M., Abaspour, S.R., Kimiaeifar, A., Rahimpour, M.: On the Analytical Solution of Kirchhoff Simplified Model for Beam by using of Homotopy Analysis Method. World Applied Sciences Journal 6(3), 297302 (2009) 


\section{Mashinchi Joubari, R. Asghari/ TJMCS Vol. 4 No. 3 (2012) 371 - 379}

[20] Ghotbi, Abdoul R., Bararnia, H., Domairry, G., Barari, A.: Investigation of a powerful analytical method into natural convection boundary layer flow. Commun.Nonlinear Sci. Numer.Simulat. 14, 2222-2228 (2009)

[21] Sohouli, A.R., Famouri, M., Kimiaeifar, A., Domairry, G.: Application of homotopy analysis method for natural convection of Darcian fluid about a vertical full cone embedded in pours media prescribed surface heat flux. Commun.Nonlinear Sci. Numer.Simulat. 15, 1691-1699 (2010)

[22] He, J.H.: Some Asymptotic Methods for Strongly Nonlinear Equations. International Journal of Modern Physics B 20(10), 1141-1199 (2006)

[23] ÖzisTurgut, YıldırımAhmet: Generating the periodic solutions for forcing van der Pol oscillators by the Iteration Perturbation method. Nonlinear Analysis: Real World Applications 10 1984-1989 (2009)

[24] YounesianDavood, KalamiYazdi Mohammad, Askari Hassan, Saadatnia Zia: Frequency Analysis of Higherorder Duffing Oscillator using Homotopy and Iteration-Perturbation Techniques. 18th Annual International Conference on Mechanical Engineering-ISME2010-3187.

[25] Lu Junfeng: An analytical approach to the Fornberg-Whitham type equations by using the variational iteration method. Computers and Mathematics with Applications 61, 2010-2013 (2011)

[26] Shang Xufeng, Han Danfu: Application of the variational iteration method for solving nth-order integrodifferential equations. Journal of Computational and Applied Mathematics 234, 1442-1447 (2010)

[27] Barari, A., Kaliji, H.D., Ghadimi, M., Domairry, G.: Non-linear vibration of Euler-Bernoulli beams. Latin American Journal of Solids and Structures 8, In Press (2011)

[28] Zhao Yongxiang, Xiao Aiguo: Variational iteration method for singular perturbation initial value problems. Computer Physics Communications 181 947-956 (2010)

[29] Joneidi A.A., Ganji D.D., Babaelahi M.: Differential Transformation Method to determine fin efficiency of convective straight fins with temperature dependent thermal conductivity. International Communications in Heat and Mass Transfer 36, 757-762 (2009)

[30] Abdel-Halim Hassan I.H.: Application to differential transformation method for solving systems of differential equations. Applied Mathematical Modeling 32, 2552-2559 (2008)

[31] Kaliji, H.D., Fereidoon, A., Ghadimi, M., Eftari, M.: Analytical Solutions for Investigating Free Vibration of Cantilever Beams. World Applied Sciences Journal (Special Issue of Applied Math) 9, 44-48, 2010.

[32] Zhao Ling: He's frequency-amplitude formulation for nonlinear oscillators with an irrational force. Computers and Mathematics with Applications 58, 2477-2479 (2009)

[33] Fereidoon, A., Ghadimi, M., Barari, A., Kaliji, H.D., Domairry, G.: Nonlinear vibration of oscillation systems using frequency-amplitude formulation. Journal of Shock and Vibration, 18, 1-10 (2011)

[34] He J.H.: Max-min approach to nonlinear oscillators. Int. J. Nonlinear Sci. Numer.Simul. 9(2), 207-210 (2008)

[35] Ibsen, L.B., Barari, A., Kimiaeifar, A.: Analysis of highly nonlinear oscillation systems using He's max-min method and comparison with homotopy analysis and energy balance methods. Sadhana 35, 433-448 (2010)

[36] Ganji, S.S., Barari, A., Ganji, D.D.: Approximate analysis of two-mass-spring systems and buckling of a column. Computers and Mathematics with Applications 61, 1088-1095 (2011)

[37] Sfahani, M.G., Ganji, S.S., Barari, A., Mirgolbabaei, H., Domairry, G.: Analytical solutions to nonlinear conservative oscillator with fifth-order nonlinearity. Earthq.Eng. \& Eng. Vib. 9, 367-374 (2010) DOI: 10.1007/s11803-010-0021-5

[38] M. Ghadimi, H.D. Kaliji, A. Barari, Analytical Solutions to Nonlinear Mechanical Oscillation Problems, Journal of Vibroengineering, 13 (2), 2011, pp. 133-143. 